\title{
Development and validation of a risk nomogram for postoperative acute kidney injury in older patients undergoing liver resection: a pilot study
}

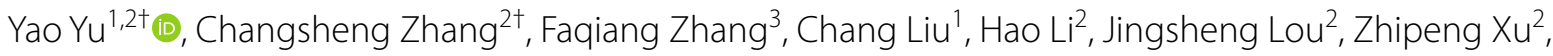
Yanhong $\mathrm{Liu}^{2}$, Jiangbei $\mathrm{CaO}^{2}$ and Weidong $\mathrm{Mi}^{1,2^{*}}$ (D)

\begin{abstract}
Background: Postoperative acute kidney injury (AKI) is associated with poor clinical outcomes. Early identification of high-risk patients of developing postoperative AKI can optimize perioperative renal management and facilitate patient survival. The present study aims to develop and validate a nomogram to predict postoperative AKI after liver resection in older patients.
\end{abstract}

Methods: A retrospective observational study was conducted involving data from 843 older patients scheduled for liver resection at a single tertiary high caseload general hospital between 2012 and 2019. The data were randomly divided into training $(70 \%, n=599)$ and validation $(30 \%, n=244)$ datasets. The training cohort was used to construct a predictive nomogram for postoperative AKI with the logistic regression model which was confirmed by a validation cohort. The model was evaluated by receiver operating characteristic (ROC) curve, calibration plot, and decision curve analysis in the validation cohort. A summary risk score was also constructed for identifying postoperative AKI patients.

Results: Postoperative AKI occurred in 155 (18.4\%) patients and was highly associated with in-hospital mortality (5.2\% vs. $0.7 \%, P<0.001$ ). The six predictors selected and assembled into the nomogram included age, preexisting chronic kidney disease (CKD), non-steroidal anti-inflammatory drugs (NSAIDs) usage, intraoperative hepatic inflow occlusion, blood loss, and transfusion. The predictive nomogram performed well in terms of discrimination with area under ROC curve (AUC) in training (0.73, 95\% confidence interval (Cl): 0.68-0.78) and validation (0.71, 95\% Cl: 0.63$0.80)$ datasets. The nomogram was well-calibrated with the Hosmer-Lemeshow chi-square value of 9.68 $(P=0.47)$. Decision curve analysis demonstrated a significant clinical benefit. The summary risk score calculated as the sum of points from the six variables (one point for each variable) performed as well as the nomogram in identifying the risk of AKI (AUC 0.71, 95\% Cl: 0.66-0.76).

Conclusion: This nomogram and summary risk score accurately predicted postoperative AKI using six clinically accessible variables, with potential application in facilitating the optimized perioperative renal management in older patients undergoing liver resection.

\footnotetext{
*Correspondence: wwdd1962@163.com

${ }^{\dagger}$ Yao Yu and Changsheng Zhang contributed equally to this work.

2 Department of Anesthesiology, The First Medical Center of Chinese PLA

General Hospital, 28th Fuxing Road, Haidian District, Beijing 100853,

China

Full list of author information is available at the end of the article
}

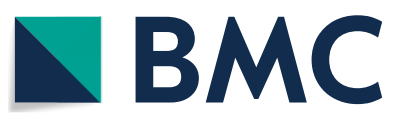

(c) The Author(s) 2022. Open Access This article is licensed under a Creative Commons Attribution 4.0 International License, which permits use, sharing, adaptation, distribution and reproduction in any medium or format, as long as you give appropriate credit to the original author(s) and the source, provide a link to the Creative Commons licence, and indicate if changes were made. The images or other third party material in this article are included in the article's Creative Commons licence, unless indicated otherwise in a credit line to the material. If material is not included in the article's Creative Commons licence and your intended use is not permitted by statutory regulation or exceeds the permitted use, you will need to obtain permission directly from the copyright holder. To view a copy of this licence, visit http://creativecommons.org/licenses/by/4.0/. The Creative Commons Public Domain Dedication waiver (http://creativeco mmons.org/publicdomain/zero/1.0/) applies to the data made available in this article, unless otherwise stated in a credit line to the data. 
Trial registration: NCT04922866, retrospectively registered on clinicaltrials.gov on June 11, 2021.

Keywords: Acute kidney injury, Hepatectomy, Elderly patients, Renal injury, Risk score, Prediction

\section{Background}

Despite the recent technical advances in surgical procedures, there have been considerably high rates of operative mortality and morbidity resulting from complex surgical procedures, especially in emergency surgery cases [1]. Among the various types of postoperative organ injuries, acute kidney injury (AKI) is particularly prevalent in 5 to $20 \%$ of patients undergoing major noncardiac surgery and in 10 to $40 \%$ of high-risk patients with hepatic resection surgery [2-5]. Previous studies have confirmed AKI as an independent contributor to peri- and postoperative morbidity and mortality in both cardiac and non-cardiac surgeries [6-9]. It has been reported that postoperative AKI could increase the risk of death by twelve folds and extend the hospital stay by five days than usual [2], and may also contribute to the development of advanced chronic kidney disease (CKD), involving substantial healthcare burden [10].

In the older population, however, the incidence of postoperative AKI is significantly higher as the filtration capacity of the kidney decreases about $1 \%$ every year after the age of 40 , even in the healthy population [11, 12]. Microstructural and functional changes in the kidney-related to healthy aging are reportedly aggravated in the presence of CKD [11]. Aging reduces renal autoregulatory capacity due to physiological and functional changes, leading to different types of kidney diseases, such as vascular sclerosis [13], declining glomerular filtration rate (GFR) [14], thereby enhancing the susceptibility of the older population to postoperative AKI. Notably, Chao et al found that almost $20 \%$ of patients in a cohort of 4000 older subjects developed postoperative AKI [15]. Moreover, postoperative AKI cases can significantly increase during certain procedures, such as cardiac and vascular surgeries, up to $30 \%$ [16]. In contrast to cardiac or vascular surgery-associated postoperative AKI incidence, there are not enough studies on hepatic resection-related AKI onset and its risk factors and prognosis.

Generally, in-depth knowledge of risk factors and identification of high-risk populations, who are predisposed to develop AKI is urgently warranted as a preventive measure to minimize operative mortality rate and to design efficient therapeutic strategies. Efficient preventive measures to reduce the risk of AKI include precise assessment of renal functions, careful administration of nephrotoxic drugs, minimizing procedural injuries, and an effective intravenous fluid regimen. However, no validated model is currently available to predict the risk of postoperative AKI in older patients following liver resection surgery. Therefore, the present study aimed to establish and validate a predictive nomogram and a simple risk score assessment to identify the risk of developing postoperative AKI in older patients following liver resection. It would be of great clinical significance for clinicians to be able to predict whether a patient would need special attention for optimal renal management after hepatectomy surgery.

\section{Methods}

This retrospective observational study was conducted using the dataset of inpatient surgeries in the First Medical Center of Chinese PLA General Hospital. The study design and data analysis were approved by the Institutional Committee for Medical Research Ethics (approval number: S2021-335-01) and registered at clinicaltrials. gov (identifier: NCT04922866). The requirement for written informed consent was waived for this retrospective study. This manuscript adheres to the Transparent Reporting of a Multivariable Prediction Model for Individual Prognosis or Diagnosis (TRIPOD) guidelines [17].

\section{Patients}

Older patients ( $\geq 65$ years) admitted to the hospital for elective hepatectomy between January 2012, and July 2019 were included in this cohort. While patients no more than 65 years old, preoperative baseline GFR $<15 \mathrm{ml} \cdot \mathrm{min}^{-1} \cdot 1.73 \mathrm{~m}^{-2}$, emergency operation, concurrent operation, laparoscopic operation, or liver transplantation surgery were excluded.

\section{Data collection}

Data acquisition from the electronic medical record (EMR) system was performed using SQL Server (Microsoft, USA). To facilitate the clinical use of predictors, we considered the inclusion of both preoperative and intraoperative parameters into the nomogram, which might closely affect renal function. From the patient record integrated management system (PRIDE 2.1.2.193, Heren Health, China), we extracted the relevant patient demographics, including age, sex, body mass index (BMI), combined hypertension, diabetes, cardiovascular and pulmonary diseases, CKD, American Society of Anesthesiologists physical score (ASA PS), and length of hospital stay. The prescribed medication regimen included nonsteroidal anti-inflammatory drugs (NSAIDs), nephrotoxic antibiotics, glucocorticoids, and preoperative and 
intraoperative diuretics. Serum biomarkers included serum creatinine, albumin, bilirubin, hemoglobin, and fasting blood glucose. From the anesthesia information management systems (DoCare 3.1.0 build 153, MEDICALSYSTEM, China), intraoperative data were retrieved, including types and duration of the surgical procedures performed and extent of liver resection, intraoperative hepatic inflow occlusion, blood pressure and infusion of vasoactive drugs, the requirement for intraoperative infusion and transfusion, and volumes of blood loss and urine output.

\section{Definitions of outcomes}

The primary outcome was postoperative AKI, defined as an absolute increase in serum creatinine level of $\geq 26.5 \mu \mathrm{mol} \cdot \mathrm{l}^{-1}$ within $48 \mathrm{~h}$ or a 1.5 -fold increase from preoperative baseline within seven days after surgery, according to the Kidney Disease: Improving Global Outcomes (KDIGO) criterion [18]. Baseline creatinine or eGFR were defined as the closest measurement before the date of operation, within 3 months. Related definitions and diagnoses included the following: hypertension defined as systolic blood pressure $\geq 140 \mathrm{mmHg}$ and/ or diastolic blood pressure $\geq 90 \mathrm{mmHg}$ on three different occasions; diabetes defined as reported history or physician-diagnosed diabetes or the presence of antidiabetic medication; cardiovascular diseases included reported history or physicians-diagnosis of coronary heart disease, angina, myocardial infarction, heart failure, or stroke; pulmonary disease defined as self-reported chronic bronchitis, chronic obstructive pulmonary disease (COPD), emphysema, interstitial lung disease (ILD), and asthma; preoperative CKD was defined as the GFR of less than $60 \mathrm{ml} \cdot \mathrm{min}^{-1} \cdot 1.73 \mathrm{~m}^{-2}$ for adults based on the CKD Epidemiology Collaboration (CKD-EPI) equation [19]. Blood transfusion was defined as any allogeneic transfusion of at least a single unit of red blood cells.

\section{Statistical analysis}

Continuous variables were reported as medians with quartiles and compared using Wilcoxon rank-sum test as they were abnormally distributed checked by Skewness-Kurtosis All test. Categorical data were reported as frequencies and percentages and compared using the chisquared test or Fisher's exact test as appropriate.

The overall 843 patients were divided into training $(n=599)$ and validation $(n=244)$ cohorts with a split ratio of 70 and $30 \%$ using randomization method. The training dataset was used to develop the prediction model in the final logistic regression. Firstly, a univariate analysis was performed using the Wilcoxon ranksum test for continuous variables and the chi-squared test for categorical variables, with postoperative AKI as the outcome. Secondly, for the independent predictors included in the model, candidate variables that were clinically relevant to AKI and $P$-value less than 0.1 in univariate analysis were included in the multivariable model. Additionally, the number of outcome events was considered, that is, at least ten outcome events per variable (EPV) generally [20]. Based on the EPV approach for determining sample size, our sample size could be expected to provide robust estimates. Thirdly, the enter methodology was applied to select predictive variables in the final model. Finally, the multivariable logistic regression model was formulated to establish the prediction model, and a nomogram was further performed to predict postoperative $\mathrm{AKI}$ in the training dataset.

The predictive performance of the model was subsequently evaluated in patients from the validation cohort. The discrimination of the nomogram was assessed by calculating the area under the receiver operating characteristic (ROC) curve. The model's calibration was evaluated using the Hosmer-Lemeshow goodness of fit test. Finally, the decision curve analysis (DCA) was performed to reveal the net benefits with each threshold probability [21]. Statistical analyses were performed using R 4.0.1 (R Foundation for Statistical Computing, Vienna, Austria) and SPSS 25.0 (IBM Corp., Chicago, IL, USA) software. Statistical significance was accepted at the 0.05 level, and all tests were two-tailed.

\section{Results \\ Patient characteristics}

A total of 930 participants were screened for eligibility from January 2012 to July 2019. After the exclusion of 77 patients who undertook combined or multiple surgeries, 853 patients were selected with liver resection. Additionally, ten subjects with missing values were excluded, thus leaving behind 843 individuals who were enrolled in the final analysis (Fig. 1). Patients were divided into training (599 patients total, 111 postoperative AKI) and validation (244 patients total, 44 postoperative AKI) cohorts, respectively. Patient characteristics and perioperative variables for the overall population are listed in Tables 1 and 2. The median (quartile) age at the time of surgery was $69(66,73)$ years, where $553(66 \%)$ patients were male, and 290 (34\%) patients were female. The median (quartile) duration of operation was $3.4(2.6,4.5) \mathrm{h}$, and the median time to discharge was $10(8,13)$ days. Thirteen $(1.5 \%)$ patients died before discharge.

Overall, 155 (18.4\%) patients developed postoperative AKI. Patients who developed postoperative AKI were at a higher risk of death (5.2\%) before discharge than those without AKI $(0.7 \%, P<0.001)$ (Additional file 1$)$. 


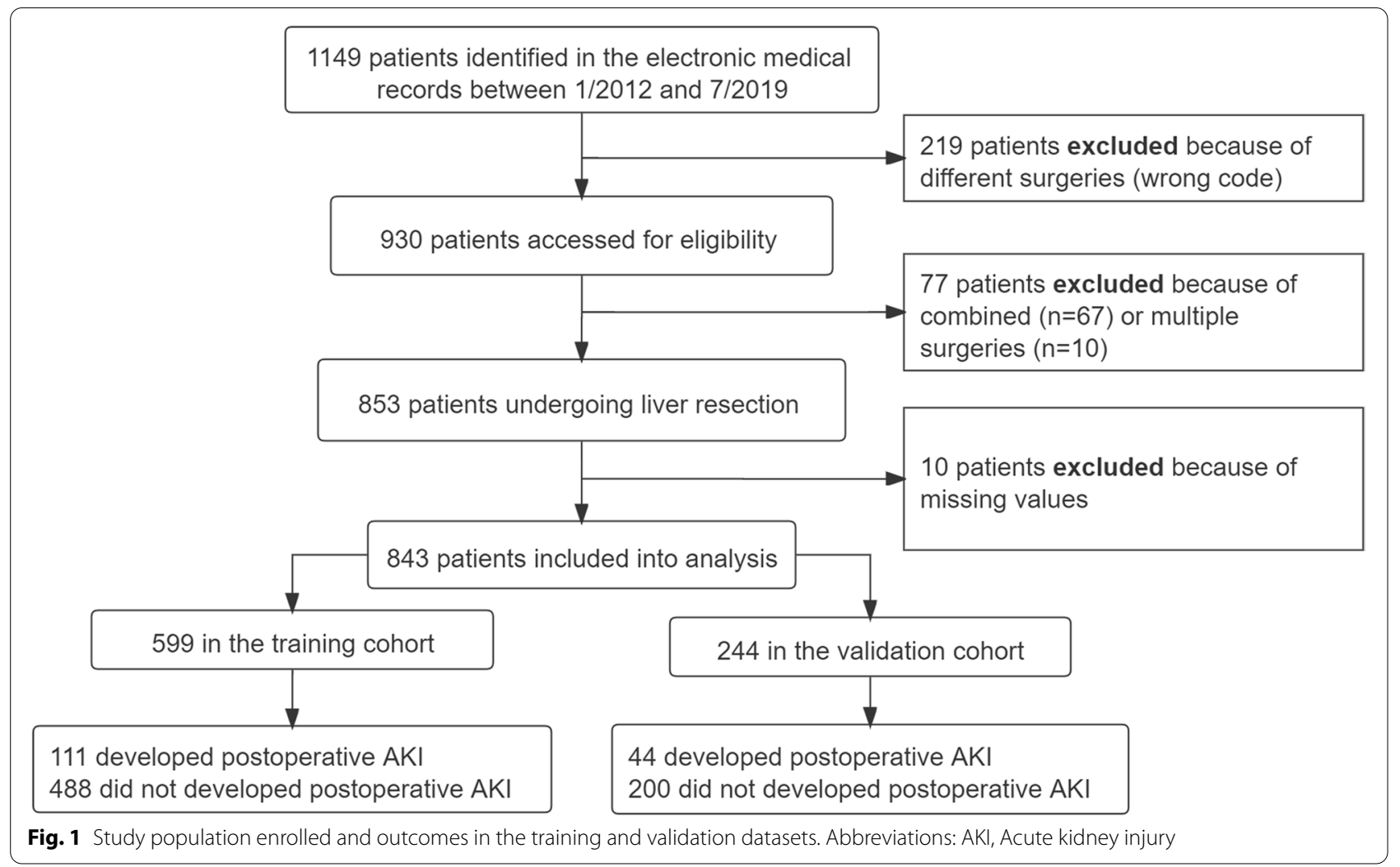

\section{Development of a predictive nomogram}

The training dataset from 599 patients was used to establish the predictive nomogram. The univariate logistic regression analysis of factors associated with postoperative AKI is summarized in Table 3. Multivariable logistic regression analysis demonstrated that the following variables were independent predictors of postoperative AKI in older patients with liver resection: age, history of CKD, hepatic inflow occlusion, intraoperative blood loss and transfusion, and perioperative use of NSAIDs (Table 4 and Additional file 2). These six predictors selected as the optimal subset for predicting postoperative AKI were incorporated into the predictive nomogram (Fig. 2). For an individual patient, find each of the six predictor's points on the top line and add them together. The total points corresponding to the bottom line indicated the percentage of probability of postoperative AKI. An online calculator based on the multivariable logistic regression model was developed to allow clinicians to enter the values of the 6 predictors required for the risk score with automatic calculation of the likelihood (with 95\% CI) that a patient will develop AKI (https://yuyao0505.shinyapps. io/DynNomapp/) (Additional file 3).

In addition, there were also results showing that univariate risk of intraoperative diuretics and vasopressors is statistically significant; while the multivariable model risk did not show statistical significance (Additional file 4). And no statistical significance of urine output and fluid balance were found in uni- and multivariable analyses (Table 3 and Additional file 4).

\section{Validation of the nomogram}

The validation dataset of the remaining 244 patients was used to evaluate the model's predictive performance. The nomogram had a satisfactory capacity with the areas under ROC curve (AUC) of 0.73 (95\% confidential interval (CI): 0.68-0.78) and 0.71 (95\% CI: 0.63-0.80) in the training and validation cohorts, respectively (Fig. 3A and B). Besides, the nomogram had a well-calibrated performance with Hosmer-Lemeshow chi-square value of 9.68 $(P=0.47)$ (Fig. 3C). The DCA showed the satisfactory net benefit that the patient could receive from the predictive nomogram, with a wide range (10 to $40 \%)$ of high-risk threshold (Fig. 3D).

\section{Summary risk score model}

To promote the clinical application of the nomogram, variables selected for the predictive model were dichotomized and counted. Continuous parameters were divided into two subgroups with good or poor prediction based on the cut-off values determined by ROC analyses. One point was assigned to 
Table 1 Patient Characteristics and baseline variables stratified by datasets

\begin{tabular}{|c|c|c|c|}
\hline Variables & $\begin{array}{l}\text { Training } \\
(n=599)\end{array}$ & $\begin{array}{l}\text { Validation } \\
(n=244)\end{array}$ & $P$ Value \\
\hline Age, years & $69(66,72)$ & $69(66,73)$ & 0.36 \\
\hline Sex (Male), n (\%) & $385(64.3)$ & $168(68.9)$ & 0.23 \\
\hline $\mathrm{BMI}, \mathrm{kg} \cdot \mathrm{m}^{-2}$ & $23.6(21.6,25.7)$ & $23.5(20.9,26.0)$ & 0.68 \\
\hline Hypertension, n (\%) & $230(38.4)$ & $89(36.5)$ & 0.64 \\
\hline Diabetes, n (\%) & $109(18.2)$ & $48(19.7)$ & 0.63 \\
\hline $\begin{array}{l}\text { Cardiovascular diseases, } \\
\text { n (\%) }\end{array}$ & $80(13.4)$ & $43(17.6)$ & 0.13 \\
\hline CKD, n (\%) & $26(4.3)$ & $6(2.5)$ & 0.24 \\
\hline Pulmonary diseases, n (\%) & $32(5.3)$ & $12(4.8)$ & 0.87 \\
\hline Hepatitis/ cirrhosis, n (\%) & $125(20.9)$ & $49(20.1)$ & 0.85 \\
\hline ASA PS, n (\%) & & & 0.77 \\
\hline$|-| \mid$ & $491(82.0)$ & $203(83.2)$ & \\
\hline III-IV & $108(18.0)$ & $41(16.8)$ & \\
\hline Pathology, n (\%) & & & 0.13 \\
\hline Hepatoma & $383(63.9)$ & $154(63.1)$ & \\
\hline Cholangiocarcinoma & $82(13.7)$ & $46(18.9)$ & \\
\hline Hepatic Metastasis & $16(2.7)$ & $8(3.3)$ & \\
\hline Benign & $118(19.7)$ & $36(14.8)$ & \\
\hline Hemoglobin, $g \cdot L^{-1}$ & $131(120,142)$ & $130(119,140)$ & 0.34 \\
\hline Albumin, $g \cdot L^{-1}$ & $39.0(36.1,41.6)$ & $39.0(36.8,41.4)$ & 0.71 \\
\hline Total bilirubin, $\mu \mathrm{mol} \cdot \mathrm{L}^{-1}$ & $13.1(9.2,19.6)$ & $12.6(9.7,19.7)$ & 1.00 \\
\hline Direct bilirubin, $\mu \mathrm{mol} \cdot \mathrm{L}^{-1}$ & $4.3(3.0,7.5)$ & $4.3(3.0,7.7)$ & 0.90 \\
\hline $\begin{array}{l}\text { Fasting blood glucose, } \\
\mu \mathrm{mol} \cdot \mathrm{L}^{-1}\end{array}$ & $5.04(4.58,5.83)$ & $5.12(4.62,5.96)$ & 0.51 \\
\hline Creatinine, $\mu \mathrm{mol} \cdot \mathrm{L}^{-1}$ & $68.4(59.0,79.2)$ & $66.1(58.5,76.8)$ & 0.29 \\
\hline eGFR, ml.min $1.73 \mathrm{~m}^{-2}$ & $89.8(80.8,94.4)$ & $90.1(84.5,95.2)$ & 0.11 \\
\hline
\end{tabular}

Notes: Continuous data are shown as medians (quartiles) and compared using Wilcoxon rank-sum test. Categorical variables are shown as frequencies (percentages) and compared using the chi-squared test or Fisher's exact test as appropriate

Abbreviations: ASA PS American Society of Anesthesiologists physical score, $B M I$ Body mass index, CKD Chronic kidney disease; eGFR, estimated glomerular filtration rate

each of the following predictors: age above 67 years, CKD history, NSAIDs medication, intraoperative hepatic inflow occlusion, intraoperative blood loss $>300 \mathrm{~mL}$, and requirement for blood transfusion (Additional files 5 and 6). The summary score for individual patients ranged from 0 to 6 . The risk of AKI ranged from $4 \%$ with a score of 1 , to $50 \%$, with a score of 6 . The summary scores were then grouped as low (0 to 2 points) and high-risk groups (3 to 6 points), as shown in Fig. 4A. AUC for predicting postoperative AKI was 0.71 (95\% CI: 0.66-0.76) for the summary score model, which was not significantly different from the previous model (Fig. 4B).
Table 2 Pre- and intraoperative variables and outcomes stratified by datasets

\begin{tabular}{|c|c|c|c|}
\hline Variables & $\begin{array}{l}\text { Training } \\
(\mathrm{n}=599)\end{array}$ & $\begin{array}{l}\text { Validation } \\
(n=244)\end{array}$ & $P$ Value \\
\hline NSAIDs, n (\%) & $540(90.2)$ & $217(88.9)$ & 0.62 \\
\hline Preoperative diuretics, n (\%) & $41(6.8)$ & $19(7.8)$ & 0.66 \\
\hline Nephrotoxic antibiotics, n (\%) & $52(8.7)$ & $27(11.1)$ & 0.30 \\
\hline Glucocorticoid, n (\%) & & & 0.75 \\
\hline Dexamethasone & $163(27.2)$ & $71(29.1)$ & \\
\hline Methylprednisolone & $277(46.2)$ & $106(43.4)$ & \\
\hline None & $159(26.5)$ & $67(27.5)$ & \\
\hline Vasoactive agents, n (\%) & & & 0.14 \\
\hline None & $326(54.4)$ & $122(50.0)$ & \\
\hline Hypertensive agents & $166(27.7)$ & 79 (32.4) & \\
\hline Hypotensive agents & $62(10.4)$ & $32(13.1)$ & \\
\hline Both & $45(7.5)$ & $11(4.5)$ & \\
\hline Intraoperative diuretics, n (\%) & $90(15.0)$ & $48(19.7)$ & 0.10 \\
\hline MAP < 60 mmHg, n (\%) & $378(63.1)$ & $169(69.3)$ & 0.10 \\
\hline $\begin{array}{l}\text { Duration of MAP }<60 \mathrm{mmHg} \text {, } \\
\text { min }\end{array}$ & $5(0,20)$ & $10(0,20)$ & 0.08 \\
\hline Duration of operation, $\mathrm{h}$ & $3.4(2.7,4.5)$ & $3.4(2.6,4.7)$ & 0.95 \\
\hline Fluid balance, $\mathrm{ml} \cdot \mathrm{kg}^{-1} \cdot \mathrm{h}^{-1}$ & $11.8(9.0,14.9)$ & $11.5(9.0,14.6)$ & 0.77 \\
\hline $\begin{array}{l}\text { Hydroxyethyl starch, } \mathrm{ml} \cdot \mathrm{kg}^{-1} \text {. } \\
\mathrm{h}^{-1}\end{array}$ & $3.6(2.6,4.9)$ & $3.6(2.7,4.9)$ & 0.89 \\
\hline Ringer's solution, $\mathrm{ml} \cdot \mathrm{kg}^{-1} \cdot \mathrm{h}^{-1}$ & $9.9(7.7,12.9)$ & $9.8(7.5,12.5)$ & 0.46 \\
\hline Urine output, $\mathrm{m} \cdot \mathrm{kg}^{-1} \cdot \mathrm{h}^{-1}$ & $1.7(1.0,2.8)$ & $1.5(0.9,2.8)$ & 0.33 \\
\hline Blood loss, $100 \mathrm{ml}$ & $3(2,6)$ & $3(2,5)$ & 0.99 \\
\hline Blood transfusion, n (\%) & $137(22.9)$ & $55(22.5)$ & 1.00 \\
\hline Resection extent, n (\%) & & & 0.55 \\
\hline Right liver & $86(14.4)$ & $34(13.9)$ & \\
\hline Left liver & $155(25.9)$ & $72(29.5)$ & \\
\hline Partial & $358(59.8)$ & $138(56.6)$ & \\
\hline Hepatic inflow occlusion, n (\%) & $362(60.4)$ & $151(61.9)$ & 0.76 \\
\hline Duration of occlusion, min & $14(0,30)$ & $14(0,27)$ & 0.82 \\
\hline LOS, days & $10(8,13)$ & $10(8,13)$ & 0.79 \\
\hline Postoperative AKI, n (\%) & $111(18.5)$ & $44(18.0)$ & 1.92 \\
\hline Death before discharge, n (\%) & $7(1.2)$ & $6(2.5)$ & 0.22 \\
\hline
\end{tabular}

Notes: "Hypertensive agents" in vasoactive agents include ephedrine, epinephrine, dopamine, norepinephrine, and phenylephrine. "Hypotensive agents" in vasoactive agents include urapidil and nicardipine. Nephrotoxic antibiotics refer to aminoglycoside and sulfonamide antibiotics. Continuous data are shown as medians (quartiles) and compared using Wilcoxon ranksum test. Categorical variables are shown as frequencies (percentages) and compared using the chi-squared test or Fisher's exact test as appropriate Abbreviations: AKI Acute kidney injury, LOS Length of hospital stay, MAP Mean arterial pressure, NSAIDs Non-steroidal anti-inflammatory drugs

\section{Discussion}

Overall, our studies established a predictive nomogram and a simple risk score assessment to identify the risk of developing postoperative AKI in older patients post liver resection. Comprehensive information from overall 843 patients with hepatic resection over the past eight years 
Table 3 Univariate logistic regression analysis of study variables vs postoperative AKI in the training cohort

\begin{tabular}{|c|c|c|c|}
\hline Variables & $\beta$ Coefficient (95\% Cl) & OR $(95 \% \mathrm{Cl})$ & $P$ Value \\
\hline Age, years & $0.05(0.01-0.10)$ & $1.05(1.01-1.10)$ & 0.02 \\
\hline Sex, female vs male & $0.11(-0.31-0.54)$ & $1.12(0.73-1.71)$ & 0.61 \\
\hline $\mathrm{BMI}, \mathrm{kg} \cdot \mathrm{m}^{-2}$ & $-0.04(-0.10-0.03)$ & $0.96(0.90-1.03)$ & 0.24 \\
\hline Hypertension, yes vs no & $0.38(-0.03-0.80)$ & $1.47(0.97-2.22)$ & 0.07 \\
\hline Diabetes, yes vs no & $0.46(-0.03-0.96)$ & $1.59(0.97-2.61)$ & 0.06 \\
\hline Cardiovascular diseases, yes vs no & $0.11(-0.48-0.70)$ & $1.11(0.62-2.01)$ & 0.72 \\
\hline$C K D$, yes vs no & $1.07(0.25-1.89)$ & $2.92(1.29-6.62)$ & 0.01 \\
\hline Pulmonary diseases, yes vs no & $-0.22(-1.19-0.76)$ & $0.80(0.30-2.14)$ & 0.66 \\
\hline Hepatitis/ cirrhosis, yes vs no & $-0.15(-0.67-0.37)$ & $0.86(0.51-1.45)$ & 0.58 \\
\hline ASA PS, III-IV vS I-II & $0.54(0.05-1.03)$ & $1.72(1.05-2.81)$ & 0.03 \\
\hline \multicolumn{4}{|l|}{ Pathology, vs hepatoma } \\
\hline Cholangiocarcinoma & $0.25(-0.32-0.82)$ & $1.28(0.72-2.27)$ & 0.40 \\
\hline Hepatic Metastasis & $0.35(-0.81-1.51)$ & $1.41(0.44-4.51)$ & 0.56 \\
\hline Benign & $-0.48(-1.08-0.12)$ & $0.62(0.34-1.12)$ & 0.12 \\
\hline Hemoglobin, $g \cdot L^{-1}$ & $-0.02(-0.03--0.01)$ & $0.98(0.97-0.99)$ & $<0.01$ \\
\hline Albumin, $g \cdot L^{-1}$ & $-0.08(-0.13--0.03)$ & $0.92(0.88-0.97)$ & $<0.01$ \\
\hline Total bilirubin, per $10 \mu \mathrm{mol} \cdot \mathrm{L}^{-1}$ & $0.04(0.002-0.07)$ & $1.04(1.00-1.07)$ & 0.04 \\
\hline Direct bilirubin, per $10 \mu \mathrm{mol} \cdot \mathrm{L}^{-1}$ & $0.05(0.01-0.09)$ & $1.05(1.01-1.09)$ & 0.02 \\
\hline Fasting blood glucose, $\mu \mathrm{mol} \cdot \mathrm{L}^{-1}$ & $0.07(-0.02-0.16)$ & $1.07(0.98-1.17)$ & 0.12 \\
\hline Creatinine, $\mu \mathrm{mol} \cdot \mathrm{L}^{-1}$ & $0.004(-0.01-0.01)$ & $1.00(0.99-1.01)$ & 0.39 \\
\hline $\mathrm{eGFR}, \mathrm{ml} \cdot \mathrm{min} \cdot 1.73 \mathrm{~m}^{-2}$ & $-0.01(-0.02-0.01)$ & $0.99(0.98-1.01)$ & 0.33 \\
\hline NSAIDs, yes vs no & $1.54(0.36-2.72)$ & $4.67(1.43-15.19)$ & 0.01 \\
\hline Preoperative diuretics, yes vs no & $0.52(-0.20-1.24)$ & $1.68(0.81-3.46)$ & 0.16 \\
\hline Nephrotoxic antibiotics, yes vs no & $0.54(-0.11-1.19)$ & $1.71(0.89-3.28)$ & 0.11 \\
\hline \multicolumn{4}{|l|}{ Glucocorticoid, vs None } \\
\hline Dexamethasone & $-0.20(-0.78-0.37)$ & $0.82(0.46-1.45)$ & 0.49 \\
\hline Methylprednisolone & $0.06(-0.43-0.56)$ & $1.06(0.65-1.75)$ & 0.80 \\
\hline \multicolumn{4}{|l|}{ Vasoactive agents, vs none } \\
\hline Hypertensive agents & $0.48(0.01-0.95)$ & $1.62(1.01-2.58)$ & 0.04 \\
\hline Hypotensive agents & $-0.57(-1.46-0.32)$ & $0.56(0.23-1.38)$ & 0.21 \\
\hline Both & $0.87(0.17-1.56)$ & $2.38(1.18-4.78)$ & 0.01 \\
\hline Intraoperative diuretic, vs no & $0.57(0.05-1.09)$ & $1.76(1.05-2.97)$ & 0.03 \\
\hline MAP $<60 \mathrm{mmHg}$, yes vs no & $0.19(-0.24-0.63)$ & $1.21(0.78-1.87)$ & 0.39 \\
\hline Duration of $\mathrm{MAP}<60 \mathrm{mmHg}$, min & $0.01(0-0.02)$ & $1.01(1.00-1.02)$ & 0.05 \\
\hline Duration of operation, $\mathrm{h}$ & $0.26(0.13-0.39)$ & $1.30(1.14-1.48)$ & $<0.01$ \\
\hline Fluid balance, $\mathrm{ml} \cdot \mathrm{kg}^{-1} \cdot \mathrm{h}^{-1}$ & $-0.002(-0.05-0.04)$ & $1.00(0.95-1.04)$ & 0.91 \\
\hline Hydroxyethyl starch, $\mathrm{ml} \cdot \mathrm{kg}^{-1} \cdot \mathrm{h}^{-1}$ & $0.07(-0.03-0.18)$ & $1.07(0.97-1.19)$ & 0.19 \\
\hline Ringer's solution, $\mathrm{ml} \cdot \mathrm{kg}^{-1} \cdot \mathrm{h}^{-1}$ & $-0.02(-0.07-0.03)$ & $0.98(0.93-1.03)$ & 0.47 \\
\hline Urine output, $\mathrm{ml} \cdot \mathrm{kg}^{-1} \cdot \mathrm{h}^{-1}$ & $0.01(-0.11-0.13)$ & $1.01(0.90-1.14)$ & 0.85 \\
\hline Blood loss, per $100 \mathrm{ml}$ & $0.10(0.06-0.14)$ & $1.10(1.06-1.15)$ & $<0.01$ \\
\hline Blood transfusion, yes vs no & $1.23(0.79-1.67)$ & $3.41(2.20-5.30)$ & $<0.01$ \\
\hline \multicolumn{4}{|l|}{ Resection extent, vs right liver } \\
\hline Left liver & $-1.44(-2.09--0.80)$ & $0.23(0.12-0.45)$ & $<0.01$ \\
\hline Partial & $-1.08(-1.60--0.56)$ & $0.34(0.20-0.57)$ & $<0.01$ \\
\hline Hepatic inflow occlusion, yes vs no & $0.48(0.04-0.92)$ & $1.61(1.04-2.51)$ & 0.03 \\
\hline Duration of occlusion, min & $0.01(-0.001-0.02)$ & $1.01(1.00-1.02)$ & 0.12 \\
\hline
\end{tabular}


Table 4 Predictors for postoperative AKI after liver resection in final multivariable logistic regression model

\begin{tabular}{llll}
\hline $\begin{array}{l}\text { Intercept and } \\
\text { variables }\end{array}$ & $\begin{array}{l}\boldsymbol{\beta} \text { Coefficient (95\% } \\
\text { Cl) }\end{array}$ & OR (95\% Cl) & $P$ Value \\
\hline Intercept & $-8.05(-11.75-$ & - & - \\
& $-4.36)$ & & \\
Age, years & $0.06(0.01-0.11)$ & $1.06(1.01-1.11)$ & 0.01 \\
Presence of CKD & $0.93(0.06-1.79)$ & $2.52(1.04-6.00)$ & 0.04 \\
Use of NSAIDs & $1.27(0.06-2.47)$ & $3.55(1.07-11.79)$ & 0.04 \\
Hepatic inflow occlu- & $0.49(0.02-0.96)$ & $1.63(1.02-2.60)$ & 0.04 \\
sion & & & \\
Blood loss, per $100 \mathrm{ml}$ & $0.06(0.02-0.11)$ & $1.07(1.02-1.12)$ & 0.01 \\
Blood transfusion & $0.79(0.24-1.33)$ & $2.20(1.28-3.80)$ & $<0.01$ \\
Area under ROC curve (AUC-ROC) & & \\
Training cohort: $0.73(0.68-0.78)$ & & \\
\multicolumn{7}{l}{ Validation cohort: $0.71(0.63-0.80)$} & &
\end{tabular}

Abbreviations: AKI Acute kidney injury, AUC Area under ROC curve, $\mathrm{Cl}$ Confidential interval, CKD Chronic kidney disease, NSAIDs Non-steroidal antiinflammatory drugs, $O R$ Odds ratio, ROC Receiver operating characteristic

was thoroughly reviewed and analyzed. The present nomogram and risk score assessment approach a landmark toward identifying strategies to reduce the prevalence of AKI in older patients following liver resection surgery.

\section{Summary of key findings}

Our study summarized the incidence rates, predictors, and mortality impact of AKI in an older population following liver resection surgery. Our findings further verified that the morbidity of postoperative AKI was high (18.4\%) among older populations, and associated with six-fold increased mortality rates compared with no occurrence of AKI, corroborating results from previous studies by Lim and Slankamenac [3, 22]. Therefore, a predictive nomogram and a simple risk score scale for postoperative AKI were developed and validated with six predictors readily available in clinical situations. The general overview of the main highlights of this study is as follows. First, we reviewed all relevant and eligible cases, acquired a few missing pieces of information. The large sample cohort allowed the development and validation of a predictive model. Second, the estimated GFR was selected as a better predictor of outcome to define preexisting renal dysfunction instead of serum creatinine level. Third, the simple summary risk score could be used to rapidly identify patients at high risk of developing AKI at the bedside without complex computation. Depending on this approach, we can help optimize decision-making in predicting and preventing postoperative renal dysfunction, and thus improve patients' short-and long-term outcomes.

\section{Factors affecting the model}

Several prediction models for postoperative AKI have been developed in noncardiac surgeries [4, 22, 23]. Although the occurrence of AKI remained high in about $20 \%$ of older patients following liver surgeries, the

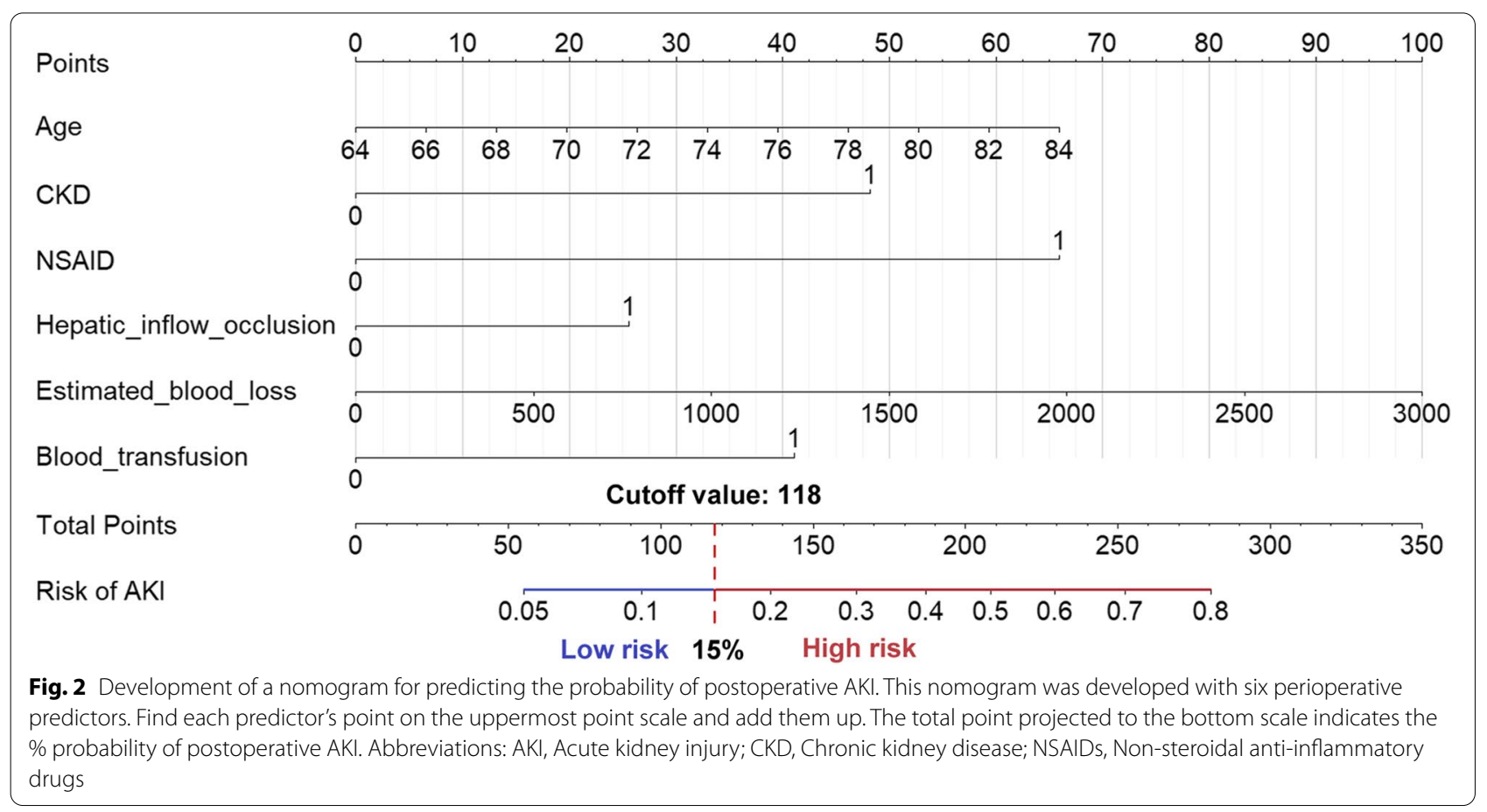



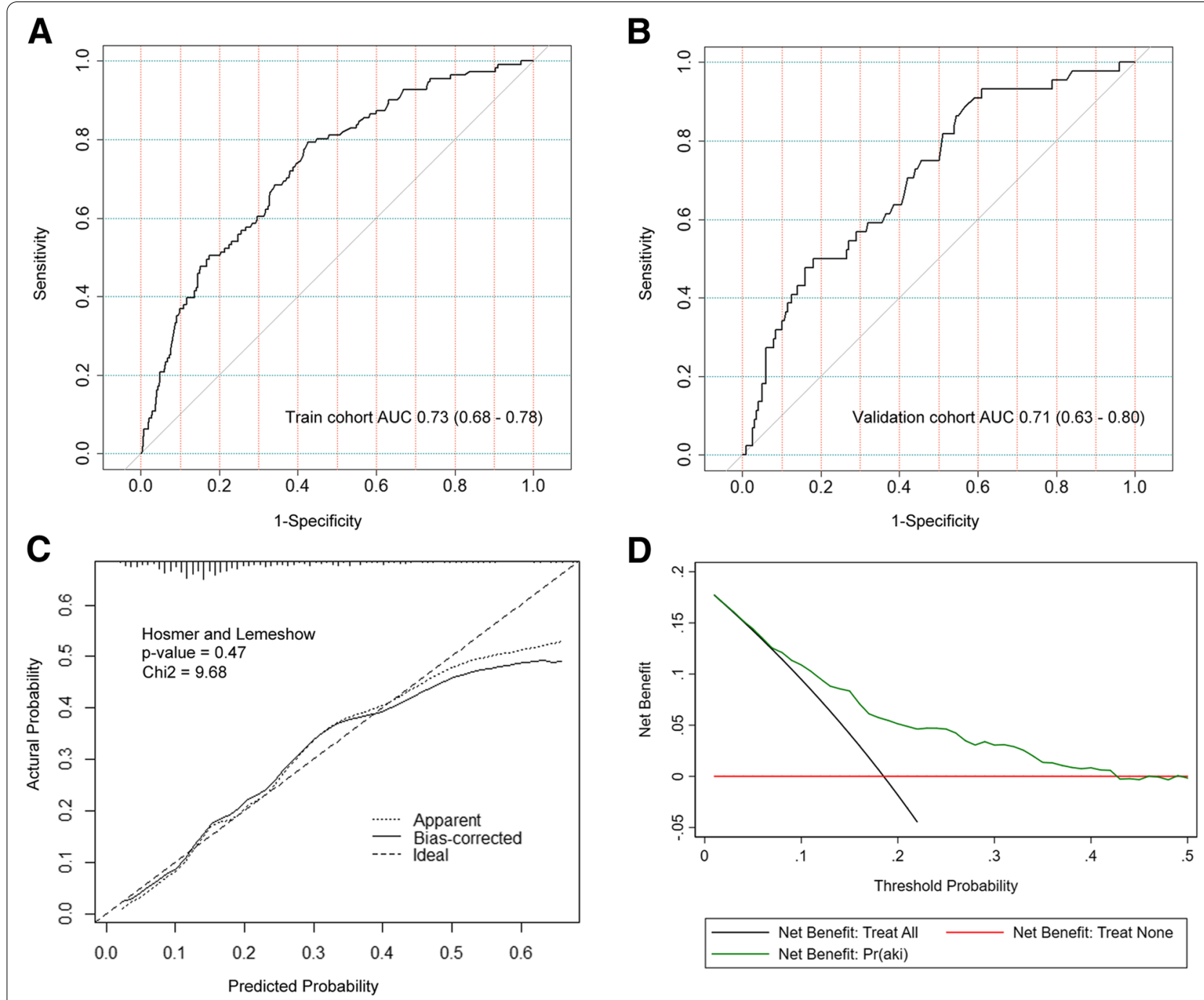

Fig. 3 Validation of the nomogram: (A) ROC curve in the training dataset; (B) ROC curve in the validation dataset; (C) Calibration curve for the training dataset; (D) Decision curve analysis for the training dataset. Abbreviations: ROC, Receiver operating characteristic

prediction of AKI in this subgroup population had been rarely concerned. Since postoperative AKI prediction can be affected by multiple factors resulting from the interactions between surgery, anesthesia procedure, and intensive care, therefore, therapeutic options to prevent and treat AKI following liver resection should involve pre-, intra-, and postoperative measures. By exploring risk factors for postoperative AKI after liver resection, we established a predictive nomogram for identifying highrisk patients that may need special attention after hepatic resection, so as to facilitate the clinical practice of early identification of such groups. Predictors identified in the present study included age, presence of CKD, NSAIDs medication, intraoperative hepatic inflow occlusion, the volume of blood loss, and transfusion. Thus, optimization of controllable factors such as preoperative renal dysfunction, NSAIDs usage, and blood transfusion due to substantial surgical bleeding may benefit patient's survival outcomes. Surgical variables such as intraoperative hepatic occlusion and blood loss remained significant predictors of postoperative AKI. A possible explanation could be the complexity of the procedures needed for hepatic inflow occlusion, the requirement of extensive hepatic excision, and severe blood loss [24, 25].

In this context, minimization of surgical trauma and bleeding were fundamental protective strategies for renal conservation and also equally crucial to restore liver function and minimize resection extent. Inflammatory responses to surgical stress and trauma lead to tubular injury and subsequent development of AKI, potentially 


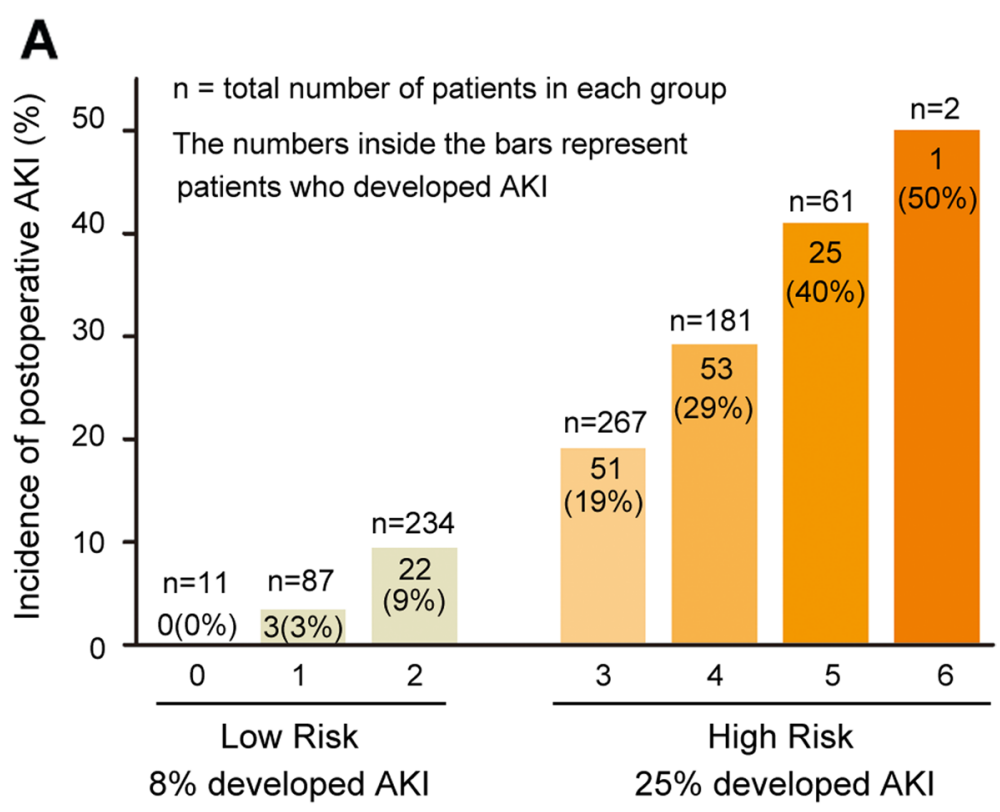

B
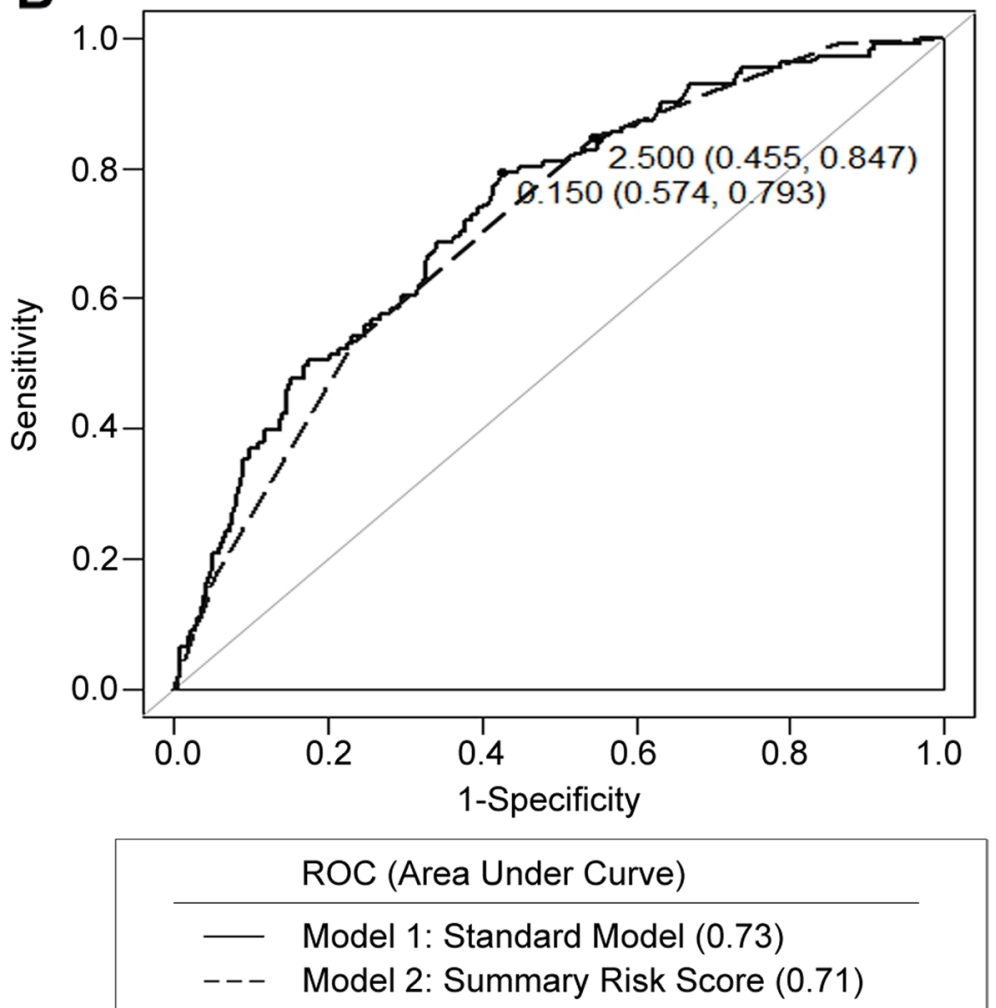

Fig. 4 A The summary risk score model for predicting postoperative AKI. One point is assigned to each of the six predictors: age above 67 years, CKD, use of NSAIDs, intraoperative hepatic inflow occlusion, intraoperative blood loss $>300 \mathrm{~mL}$, and blood transfusion. B Comparison between AUC-ROC of the multivariable logistic regression model and summary risk score model. Abbreviations: AKl, Acute kidney injury; AUC, Area under the ROC curve; CKD, Chronic kidney disease; NSAIDs, Non-steroidal anti-inflammatory drugs; ROC, Receiver operating characteristic 
due to microcirculatory dysfunction, oxidative stress, and endothelial cell injury [26]. This group of patients should be given special attention because of their exceptionally high-risk exposure to perioperative procedures. Reducing the duration of hepatic occlusion has been shown to be favorable to control trauma and alleviate ischemiareperfusion injury [27], which might contribute to the risk of renal injury mediated by inflammatory mediators and microcirculatory dysfunction [28].

Previous studies have suggested CKD as the most important patient-related risk factor [11]. It was not a new finding regarding the associations between CKD and postoperative AKI and death. While Chaudery et al has illustrated that in patients with a history of CKD, low estimated GFR does not markedly increase the mortality in the absence of AKI [8], highlighting AKI is likely to be a pivotal event connecting preoperative CKD and survival outcomes. In addition, NSAIDs inhibit cyclooxygenase activity and reduce prostaglandin secretion, resulting in tubular toxicity, renal vasoconstriction, decreasing renal blood flow, and low GFR [29, 30]. A systemic review conducted by Lee et al. has demonstrated that NSAIDs may cause a clinically transient reduction in renal function in the early postoperative period in patients with normal preoperative renal function [31]. Therefore, it is reasonable to minimize the nephrotoxic NSAID exposure in older patients concerning postoperative renal function, especially in the case of those with persistent renal dysfunction.

\section{Factors optimizing the model}

Low central venous pressure (CVP) was required during the hepatectomy to reduce bleeding complications and mortality. Hughes has consolidated reduced blood loss and transfusion requirement by maintaining CVP below $5 \mathrm{mmHg}$, by intravenous fluid restriction, and by applying diuretics [32]. At the same time, the resultant hypovolemia and oliguria may increase the risks of renal impairment, as demonstrated by Myles in a study comparing restrictive and liberal fluid administration [5]. Historically, whether restrictive or liberal fluid therapy benefits outcomes remained controversial. Surgeons expect a very low CVP, while anesthesiologists argue against the low fluid load. The prolonged fluid restriction may lead to possible renal hypoperfusion with resultant oliguria. Vasopressors to correct hypovolemia could further aggravate oliguria. Therefore, the urine output criterion may result in an overestimation of AKI [33]. It has been suggested that intraoperative urine output of $0.3 \mathrm{ml} \cdot \mathrm{kg}^{-1} \cdot \mathrm{h}^{-1}$ was the optimal threshold for oliguria, nevertheless a moderate association and poor predictive value for postoperative AKI [34-36]. Numerous studies have established the negative bearing of diuretics usage in AKI in major abdominal surgeries [37]. In the present study, we found no significant difference in urine output between patients with and without AKI; postoperative AKI was associated with more employment of diuretics, which however did not increase the model's predictive ability. The relationship between the predictive value of diuretics and urine output in AKI remained to discuss, and further studies are needed to confirm the associations, so as to promote potential targets for optimizing renal function in prospective clinical trials.

\section{Study limitations}

There are some limitations in this study. First, the study aimed to evaluate the independent risk factors predicting postoperative AKI in older patients with liver resection. Therefore, the predictors involved were commonly available in clinical settings and did not include complex frailty and comorbidity index, which represent the risk associated with perioperative mortality. Second, although intraoperative factors affecting renal function were incorporated into the model, the target mean arterial pressure (MAP) during surgery and fluid management strategies were not available in this retrospective analysis, the surrogate indicators did not obviously increase the model's predictive performance. This special attention will require further study and in combination with emerging biomarkers and novel oxygenation monitoring, clinical risk predictive models may aid in future investigations of effective prevention and treatment strategies. Third, data were derived from a retrospective single-center cohort. The reproducibility and generalization of the model in other populations are unknown. Before the model is implemented in clinical applications, it is essential to be externally validated in open, prospective multicenter studies.

\section{Conclusions}

A nomogram and risk scoring system that accurately predicts postoperative AKI in older patients undergoing liver resection were established in this study. This model considered AKI based on six conveniently available variables in clinical conditions. It could provide guidance for both clinical decision-making and scheduling a tighter perioperative follow-up in AKI high-risk patients.

\section{Abbreviations}

AKI: Acute kidney injury; ASA PS: American Society of Anesthesiologists physical score; AUC: Areas under the ROC curve; CKD: Chronic kidney disease; CKD-EPI: CKD Epidemiology Collaboration; CVP: Central venous pressure; DCA: Decision curve analysis; eGFR: estimated glomerular filtration rate; EMR: Electronic medical record; EPV: Events per variable; LOS: Length of hospital stay; MAP: Mean arterial pressure; KDIGO: Kidney Disease: Improving Global Outcomes; NSAIDs: Non-steroidal anti-inflammatory drugs; ROC: Receiver operating characteristic; TRIPOD: Transparent Reporting of a Multivariable Prediction Model for Individual Prognosis or Diagnosis. 


\section{Supplementary Information}

The online version contains supplementary material available at https://doi. org/10.1186/s12871-022-01566-z.

Additional file 1. Patient characteristics and perioperative variables stratified by AKI. Notes: "Vasopressors" in vasoactive agents include ephedrine, epinephrine, dopamine, norepinephrine, and phenylephrine. "Vasodilators" in vasoactive agents include urapidil and nicardipine. Nephrotoxic antibiotics refers to aminoglycoside and sulfonamide antibiotics. Continuous data are shown as medians (quartiles) and compared using Wilcoxon ranksum test. Categorical variables are shown as frequencies (percentages) and compared using chi-squared test or Fisher's exact test as appropriate. Abbreviations: AKI, Acute kidney injury; ASA PS: American Society of Anesthesiologists physical score; BMI, Body mass index; $\mathrm{Cl}$, Confidential interval; CKD, Chronic kidney disease; eGFR, estimated glomerular filtration rate; LOS, Length of hospital stay; MAP, Mean arterial pressure; NSAIDs, Non-steroidal anti-inflammatory drugs; OR, Odds ratio.

Additional file 2. Graphical representation of the regression model with confidence intervals. Abbreviations: NSAIDs, Non-steroidal anti-inflammatory drugs.

Additional file 3. The online web-based calculator for predicting acute kidney injury among older patients with liver resection surgery. https:// yuyao0505.shinyapps.io/DynNomapp. Abbreviations: CKD, Chronic kidney disease; NSAIDs, Non-steroidal anti-inflammatory drugs.

Additional file 4. Predictors for AKI in multivariable logistic regression model including intraoperative diuretics, urine output, vasopressors, and fluid balance. Notes: "Vasopressors" in vasoactive agents include ephedrine, epinephrine, dopamine, norepinephrine, and phenylephrine. Abbreviations: $\mathrm{Cl}$, Confidential interval; CKD, Chronic kidney disease; NSAIDs, Non-steroidal anti-inflammatory drugs; OR, Odds ratio.

Additional file 5. Summary risk score model and sensitivity/specificity values of the predictors for AKI. Abbreviations: AUC, Area under ROC curve; CKD, Chronic kidney disease; NSAIDs, Non-steroidal anti-inflammatory drugs; ROC, Receiver operating characteristic.

Additional file 6 . Sensitivity and specificity values of risk score model.

\section{Acknowledgements}

The authors thank Lei Mo and Changsong Leng of Shanghai Lejiu Healthcare Technology for their technical assistance in data extraction and statistical analyses.

\section{Authors' contributions}

YY and $C Z$ designed the study, analyzed and interpreted data, and wrote the main manuscript text. FZ collected and analyzed data. CL searched the literature, collected the data, and prepared the manuscript. $\mathrm{HL}, J \mathrm{~L}, \mathrm{ZX}$ helped design the study, analyze and interpret data. YL and JC searched the literature, designed the study, and revised the manuscript. WM designed the study, analyzed and interpreted data, prepared and revised the manuscript. All authors have read and approved the manuscript.

\section{Funding}

This research was supported by the National Key Research and Development Program of China to Weidong Mi [grant number: 2018YFC2001900] and did not receive any grant from funding agencies in the public or commercial sectors.

\section{Availability of data and materials}

The datasets analyzed during the current study are available from the corresponding author on reasonable request.

\section{Declarations}

Ethics approval and consent to participate

This study was approved by the ethics committee of Chinese PLA general hospital with approval number S2021-335-01 and registered at clinicaltrials.gov with identifier NCT04922866. The requirement for written informed consent was waived for this retrospective study. All methods were carried out in accordance with the Declaration of Helsinki.

\section{Consent for publication}

Not applicable.

\section{Competing interests}

The authors declare that they have no competing interests.

\section{Author details}

${ }^{1}$ Medical School of Chinese PLA, 28th Fuxing Road, Haidian District, Beijing 100853, China. ${ }^{2}$ Department of Anesthesiology, The First Medical Center of Chinese PLA General Hospital, 28th Fuxing Road, Haidian District, Beijing 100853, China. ${ }^{3}$ Medical College of Nankai University, 94th Weijin Road, Nankai District, Tianjin 300074, China.

Received: 4 October 2021 Accepted: 6 January 2022

Published online: 13 January 2022

\section{References}

1. Bartels K, Karhausen J, Clambey ET, Grenz A, Eltzschig HK. Perioperative organ injury. Anesthesiology. 2013;119(6):1474-89.

2. O'Connor ME, Kirwan CJ, Pearse RM, Prowle JR. Incidence and associations of acute kidney injury after major abdominal surgery. Intensive Care Med. 2016;42(4):521-30.

3. Lim C, Audureau E, Salloum C, Levesque E, Lahat E, Merle JC, et al. Acute kidney injury following hepatectomy for hepatocellular carcinoma: incidence, risk factors and prognostic value. HPB (Oxford). 2016;18(6):540-8.

4. Slankamenac K, Beck-Schimmer B, Breitenstein S, Puhan MA, Clavien P-A. Novel prediction score including pre- and intraoperative parameters best predicts acute kidney injury after liver surgery. World J Surg. 2013;37(11):2618-28.

5. Myles PS, Bellomo R, Corcoran T, Forbes A, Peyton P, Story D, et al. Restrictive versus Liberal fluid therapy for major abdominal surgery. N Engl J Med. 2018;378(24):2263-74.

6. Kork F, Balzer F, Spies CD, Wernecke K-D, Ginde AA, Jankowski J, et al. Minor postoperative increases of creatinine are associated with higher mortality and longer hospital length of stay in surgical patients. Anesthesiology. 2015;123(6):1301-11.

7. O'Connor ME, Hewson RW, Kirwan CJ, Ackland GL, Pearse RM, Prowle JR. Acute kidney injury and mortality 1 year after major non-cardiac surgery. Br J Surg. 2017;104(7):868-76

8. Chaudery H, MacDonald N, Ahmad T, Chandra S, Tantri A, Sivasakthi V, et al. Acute kidney injury and risk of death after elective surgery: prospective analysis of data from an international cohort study. Anesth Analg. 2019;128(5):1022-9.

9. Cho E, Kim S-C, Kim M-G, Jo S-K, Cho W-Y, Kim H-K. The incidence and risk factors of acute kidney injury after hepatobiliary surgery: a prospective observational study. BMC Nephrol. 2014;15:169.

10. Kashani K, Shao M, Li G, Williams AW, Rule AD, Kremers WK, et al. No increase in the incidence of acute kidney injury in a population-based annual temporal trends epidemiology study. Kidney Int. 2017;92(3):721-8.

11. Anderson S, Eldadah B, Halter JB, Hazzard WR, Himmelfarb J, Horne FM, et al. Acute kidney injury in older adults. J Am Soc Nephrol. 2011;22(1):28-38

12. Mårtensson J, Bellomo R. Perioperative renal failure in elderly patients. Curr Opin Anaesthesiol. 2015;28(2):123-30.

13. Rule AD, Amer H, Cornell LD, Taler SJ, Cosio FG, Kremers WK, et al. The association between age and nephrosclerosis on renal biopsy among healthy adults. Ann Intern Med. 2010;152(9):561-7.

14. Cowen LE, Hodak SP, Verbalis JG. Age-associated abnormalities of water homeostasis. Endocrinol Metab Clin N Am. 2013;42(2):349-70.

15. Chao C-T, Lin Y-F, Tsai H-B, Wu V-C, Ko W-J. Acute kidney injury network staging in geriatric postoperative acute kidney injury patients: shortcomings and improvements. J Am Coll Surg. 2013;217(2):240-50.

16. Nadim MK, Forni LG, Bihorac A, Hobson C, Koyner JL, Shaw A, et al. Cardiac and Vascular Surgery-Associated Acute Kidney Injury: The 20th International Consensus Conference of the ADQI (Acute Disease Quality Initiative) Group. J Am Heart Assoc. 2018;7(11):e008834. 
17. Moons KGM, Altman DG, Reitsma JB, loannidis JPA, Macaskill P, Steyerberg EW, et al. Transparent reporting of a multivariable prediction model for individual prognosis or diagnosis (TRIPOD): explanation and elaboration. Ann Intern Med. 2015;162(1):W1-73.

18. Kellum JA, Lameire N. Diagnosis, evaluation, and management of acute kidney injury: a KDIGO summary (part 1). Crit Care. 2013;17(1):204.

19. Levey AS, Stevens LA, Schmid CH, Zhang YL, Castro AF, Feldman HI, et al. A new equation to estimate glomerular filtration rate. Ann Intern Med. 2009;150(9):604-12.

20. Peduzzi P, Concato J, Kemper E, Holford TR, Feinstein AR. A simulation study of the number of events per variable in logistic regression analysis. J Clin Epidemiol. 1996;49(12):1373-9.

21. Vickers AJ, Elkin EB. Decision curve analysis: a novel method for evaluating prediction models. Med Decis Mak. 2006;26(6):565-74.

22. Slankamenac K, Breitenstein S, Held U, Beck-Schimmer B, Puhan MA, Clavien P-A. Development and validation of a prediction score for postoperative acute renal failure following liver resection. Ann Surg. 2009;250(5):720-8.

23. Kheterpal S, Tremper KK, Heung M, Rosenberg AL, Englesbe M, Shanks AM, et al. Development and validation of an acute kidney injury risk index for patients undergoing general surgery: results from a national data set. Anesthesiology. 2009;110(3):505-15.

24. Wei AC, Tung-Ping Poon R, Fan ST, Wong J. Risk factors for perioperative morbidity and mortality after extended hepatectomy for hepatocellular carcinoma. Br J Surg. 2003;90(1):33-41.

25. Tomozawa A, Ishikawa S, Shiota N, Cholvisudhi P, Makita K. Perioperative risk factors for acute kidney injury after liver resection surgery: an historical cohort study. Can J Anaesth. 2015;62(7):753-61.

26. Meersch M, Schmidt C, Zarbock A. Perioperative acute kidney injury: an under-recognized problem. Anesth Analg. 2017;125(4):1223-32.

27. Clavien P-A, Selzner M, Rüdiger HA, Graf R, Kadry Z, Rousson V, et al. A prospective randomized study in 100 consecutive patients undergoing major liver resection with versus without ischemic preconditioning. Ann Surg. 2003;238(6):843-50.

28. Jiménez-Castro MB, Cornide-Petronio ME, Gracia-Sancho J, Peralta C. Inflammasome-Mediated Inflammation in Liver Ischemia-Reperfusion Injury. Cells. 2019;8(10):1131.

29. Griffin MR, Yared A, Ray WA. Nonsteroidal antiinflammatory drugs and acute renal failure in elderly persons. Am J Epidemiol. 2000;151(5):488-96.

30. Lafrance J-P, Miller DR. Selective and non-selective non-steroidal antiinflammatory drugs and the risk of acute kidney injury. Pharmacoepidemiol Drug Saf. 2009;18(10):923-31.

31. Wick EC, Grant MC, Wu CL. Postoperative multimodal analgesia pain management with nonopioid analgesics and techniques: a review. JAMA Surg. 2017;152(7):691-7.

32. Hughes MJ, Ventham NT, Harrison EM, Wigmore SJ. Central venous pressure and liver resection: a systematic review and meta-analysis. HPB (Oxford). 2015;17(10):863-71.

33. Bressan AK, James MT, Dixon E, Bathe OF, Sutherland FR, Ball CG. Acute kidney injury following resection of hepatocellular carcinoma: prognostic value of the acute kidney injury network criteria. Can I Surg. 2018;61(5):E11-6.

34. Myles PS, Mcllroy DR, Bellomo R, Wallace S. Importance of intraoperative oliguria during major abdominal surgery: findings of the restrictive versus Liberal fluid therapy in major abdominal surgery trial. Br J Anaesth. 2019;122(6):726-33.

35. Mizota T, Yamamoto Y, Hamada M, Matsukawa S, Shimizu S, Kai S. Intraoperative oliguria predicts acute kidney injury after major abdominal surgery. Br J Anaesth. 2017;119(6):1127-34.

36. Zhao B-C, Lei S-H, Yang X, Zhang Y, Qiu S-D, Liu W-F, et al. Assessment of prognostic value of intraoperative oliguria for postoperative acute kidney injury: a retrospective cohort study. Br J Anaesth. 2021;126(4):799-807.

37. Gameiro J, Fonseca JA, Neves M, Jorge S, Lopes JA. Acute kidney injury in major abdominal surgery: incidence, risk factors, pathogenesis and outcomes. Ann Intensive Care. 2018;8(1):22.

\section{Publisher's Note}

Springer Nature remains neutral with regard to jurisdictional claims in published maps and institutional affiliations.

Ready to submit your research? Choose BMC and benefit from:

- fast, convenient online submission

- thorough peer review by experienced researchers in your field

- rapid publication on acceptance

- support for research data, including large and complex data types

- gold Open Access which fosters wider collaboration and increased citations

- maximum visibility for your research: over $100 \mathrm{M}$ website views per year

At BMC, research is always in progress.

Learn more biomedcentral.com/submissions 